\title{
Primary versus Secondary Sjögren Syndrome: Is It Time To Reconsider These Terms?
}

Classification of systemic autoimmune diseases on the basis of clinical, serological, and genetic characteristics has long been considered a necessary approach to determine disease prognosis and institute appropriate therapeutic strategies ${ }^{1}$.

Has this, however, truly been pursued by the scientific community? Probably not, because similar disease-modifying agents and several immunosuppressants are implemented as baseline therapy for combating arthritis, pleurisy, or nephritis, irrespective of the character of the underlying disease. Despite our better understanding of molecular pathobiology, which eventually led to development of targeted therapies, seropositive and seronegative arthritis are still similarly treated with tumor necrosis factor inhibitors, while certain vasculitic syndromes are managed with B cell depletion therapy in the same way as rheumatoid arthritis (RA). The responses, even to those new therapies, are not uniform because these syndromes are highly polymorphic in both clinical phenotypes and underlying pathogenetic pathways.

A prototype autoimmune disorder characterized by a wide spectrum of clinical, serological, and genetic features is Sjögren syndrome (SS). It may extend from sicca symptoms and complications of mucosal dryness as a result of exocrine gland involvement, to a systemic disease or to malignant B cell lymphoproliferation in about one-fourth and one-tenth of patients, respectively. Nevertheless, sicca manifestations may also occur in several other systemic autoimmune disorders at variable frequencies ranging from $8.3 \%$ in systemic lupus erythematosus (SLE) and 20.5\% in systemic sclerosis (SSc) to $31 \%$ in patients with $\mathrm{RA}^{2,3}$.

Thirty-five years ago, taking into account clinical, serological, and genetic characteristics of patients presenting with sicca-related manifestations either alone or in the context of RA, we coined the terms primary and secondary SS, respectively ${ }^{4}$. More specifically, it was shown that patients with SS alone presented more frequently with recurrent parotid gland enlargement, Raynaud phenomenon, purpura, lymphadenopathy, myositis, and renal involvement compared to those with SS in an RA background (RA/SS) ${ }^{5}$. The latter group was also shown to be older compared to the primary SS group and to display more frequently severe arthritis, anemia, lung involvement, presence of antibodies to citrullinated antigens, and radiographic joint damage ${ }^{6,7}$. Additionally, patients with SS alone exhibited increased frequency of anti-Ro/SSA and La/SSB autoantibodies along with HLA-B8 and HLA-DW3 alleles compared to patients with RA and sicca manifestations in which HLA-DR4 allele prevailed ${ }^{4}$. It should, however, be taken into account that these observations were based on studies of a patient population from a tertiary referral center, in which disease limited to the glandular compartment was underrepresented. Immunohistochemical studies comparing the composition of lymphocytic infiltrates in labial minor salivary glands between RA patients with sicca symptoms and SS patients alone revealed milder lesions, together with increased prevalence of dendritic cells and lower prevalence of CD4+ cells in the RA-sicca subtype, further supporting the distinction into primary and secondary forms of $\mathrm{SS}^{8}$.

Similar observations were made in patients presenting with sicca symptomatology in the context of primary biliary cirrhosis (PBC). As in patients with RA/SS, anti Ro/SSA and anti-La/SSB autoantibodies were very infrequently detected in patients with $\mathrm{PBC}$-sicca and objective signs of oral or ocular dryness, such as decreased parotid flow rates or corneal ulceration, were virtually absent. These findings along with similar immunogenetic features with RA/SS lead to the assumption that SS in PBC shares characteristics of $\mathrm{SS}$ in the context of $\mathrm{RA}^{9}$.

In contrast, ensuing studies of the clinical, serologic, and immunogenetic characteristics of patients with SLE presenting with sicca manifestations revealed similarities between SS and SLE/SS. More specifically, SLE/SS and primary SS were shown to have similar prevalence of oral/ocular dryness and of anti-Ro/SSA and anti-La/SSB autoantibody positivity, as well as similar range-of-focus scores in minor salivary gland biopsies. In contrast, in patients with SLE/SS, there was more prevalence of Raynaud phenomenon, arthritis, and serositis compared to the primary SS group. As opposed to RA, in which SS is rather a late sequelae, diagnosis of SS usually precedes that of SLE in almost two-thirds of patients. These observations, coupled with the comparable frequencies of the DRB $1 * 0301$ allele between these 2 groups of patients, suggested the presence of shared underlying pathogenetic pathways ${ }^{10}$. Moreover, studies by Drosos, et al and Salliot, et al revealed similar rates of subjective and objective sicca features, as well as comparable frequencies in anti-Ro/SSA and anti-La/SSB autoantibodies and histopathological scores in patients with $\mathrm{SSc} / \mathrm{SS}$ and patients with $\mathrm{SS}^{11,12}$.

Therefore, while the term secondary Sjögren syndrome was initially introduced to encompass patients with sicca symptoms in the context of RA, based on the distinctive character of the 2 entities, it was erroneously expanded to include other systemic autoimmune rheumatic diseases such

Personal non-commercial use only. The Journal of Rheumatology Copyright (C) 2019. All rights reserved. 
as SLE and $\mathrm{SSc}^{4}$, in which significant similarities in both clinical and immunologic and genetic terms were observed, as highlighted above. Of interest, with no further studies, the distinction between primary and secondary forms of the syndrome was also adopted in both European and European-American classification criteria for $\mathrm{SS}^{13}$. Moreover, whether overlap autoimmune disorders such as SS with autoimmune thyroiditis ${ }^{14}$, gastritis ${ }^{15}$, autoimmune cholangitis ${ }^{16}$, or anticentromere antibodies ${ }^{17}$ can be classified as primary, secondary, or associated also remains unresolved.

It then becomes evident that both primary and secondary types of SS include patients from localized disease confined mainly to the exocrine glands (local SS, RA/SS, $\mathrm{PBC} / \mathrm{SS}$ ) to extraglandular disease (systemic SS, SS/SLE, SS/Scl) affecting virtually any organ system, and to a distinct but significant group at risk for lymphoma development ${ }^{18}$. Regarding the latter complication, data from the InterLymph consortium revealed an increased risk for lymphoma development in secondary compared to primary forms of disease (OR 7.92 vs 2.84 at 2 to 5 years of followup) ${ }^{18}$.

On this basis, splitting patients into "primary" and "secondary" fails to fully reveal the wide clinical spectrum of the syndrome. Thus, in line with previous suggestions by other investigators ${ }^{12,19}$, we propose that the terms primary and secondary SS be replaced by a more descriptive terminology: SS when the disease is expressed as an entity alone or SS associated with systemic or organ-specific autoimmune diseases, provided that in all cases, the recently published set of criteria for SS are fulfilled ${ }^{20}$. Indeed, in the latter, the term secondary no longer exists, and the presence of an underlying autoimmune disease does not exclude the classification of primary SS, once the proposed criteria are fulfilled ${ }^{20}$.

\section{CLIO P. MAVRAGANI, MD,}

Associate Professor,

Departments of Physiology and Pathophysiology,

School of Medicine,

National and Kapodistrian

University of Athens:

\section{HARALAMPOS M. MOUTSOPOULOS,}

MD, FACP, FRCP (hc), Master ACR,

Department of Pathophysiology, School of Medicine,

National and Kapodistrian

University of Athens, and Academy of Athens, Athens, Greece.

Address correspondence to Dr. C.P. Mavaragani, Department of

Physiology, 75 Mikras Asias St., 11527 Athens, Greece.

E-mail:kmauragan@med.uoa.gr

\section{REFERENCES}

1. Moutsopoulos HM, Manoussakis MN. Lumping or splitting autoimmune rheumatic disorders? Lessons from Sjogren's syndrome. Br J Rheumatol 1998;37:1263-4.

2. Andonopoulos AP, Skopouli FN, Dimou GS, Drosos AA, Moutsopoulos HM. Sjogren's syndrome in systemic lupus erythematosus. J Rheumatol 1990;17:201-4.

3. Andonopoulos AP, Drosos AA, Skopouli FN, Moutsopoulos HM Sjogren's syndrome in rheumatoid arthritis and progressive systemic sclerosis. A comparative study. Clin Exp Rheumatol 1989;7:203-5.

4. Moutsopoulos HM, Chused TM, Mann DL, Klippel JH, Fauci AS, Frank MM, et al. Sjogren's syndrome (sicca syndrome): Current issues. Ann Intern Med 1980;92:212-26.

5. Moutsopoulos HM, Webber BL, Vlagopoulos TP, Chused TM, Decker JL. Differences in the clinical manifestations of sicca syndrome in the presence and absence of rheumatoid arthritis. Am J Med 1979;66:733-6.

6. Brown LE, Frits ML, Iannaccone CK, Weinblatt ME, Shadick NA, Liao KP. Clinical characteristics of RA patients with secondary SS and association with joint damage. Rheumatology 2015;54:816-20.

7. He J, Ding Y, Feng M, Guo J, Sun X, Zhao J, et al. Characteristics of sjogren's syndrome in rheumatoid arthritis. Rheumatology 2013;52:1084-9.

8. Fragoulis GE, Fragkioudaki S, Reilly JH, Kerr SC, McInnes IB, Moutsopoulos HM. Analysis of the cell populations composing the mononuclear cell infiltrates in the labial minor salivary glands from patients with rheumatoid arthritis and sicca syndrome. J Autoimmun 2016;73:85-91.

9. Tsianos EV, Hoofnagle JH, Fox PC, Alspaugh M, Jones EA, Schafer $\mathrm{DF}$, et al. Sjogren's syndrome in patients with primary biliary cirrhosis. Hepatology 1990;11:730-4.

10. Manoussakis MN, Georgopoulou C, Zintzaras E, Spyropoulou M, Stavropoulou A, Skopouli FN, et al. Sjogren's syndrome associated with systemic lupus erythematosus: Clinical and laboratory profiles and comparison with primary Sjogren's syndrome. Arthritis Rheum 2004;50:882-91.

11. Drosos AA, Andonopoulos AP, Costopoulos JS, Stavropoulos ED, Papadimitriou CS, Moutsopoulos HM. Sjogren's syndrome in progressive systemic sclerosis. J Rheumatol 1988;15:965-8.

12. Salliot C, Mouthon L, Ardizzone M, Sibilia J, Guillevin L, Gottenberg JE, et al. Sjogren's syndrome is associated with and not secondary to systemic sclerosis. Rheumatology 2007;46:321-6.

13. Vitali C, Bombardieri S, Jonsson R, Moutsopoulos HM, Alexander EL, Carsons SE, et al. Classification criteria for Sjogren's syndrome: A revised version of the European criteria proposed by the American-European Consensus Group. Ann Rheum Dis 2002;61:554-8.

14. Perez B, Kraus A, Lopez G, Cifuentes M, Alarcon-Segovia D. Autoimmune thyroid disease in primary Sjogren's syndrome. Am J Med 1995;99:480-4

15. Ebert EC. Gastrointestinal and hepatic manifestations of Sjogren syndrome. J Clin Gastroenterol 2012;46:25-30.

16. Skopouli FN, Barbatis C, Moutsopoulos HM. Liver involvement in primary Sjogren's syndrome. Br J Rheumatol 1994;33:745-8.

17. Bournia VK, Diamanti KD, Vlachoyiannopoulos PG, Moutsopoulos HM. Anticentromere antibody positive Sjogren's syndrome: A retrospective descriptive analysis. Arthritis Res Ther 2010;12:R47.

18. Smedby KE, Hjalgrim H, Askling J, Chang ET, Gregersen H, Porwit-MacDonald A, et al. Autoimmune and chronic inflammatory disorders and risk of non-Hodgkin lymphoma by subtype. J Natl Cancer Inst 2006;98:51-60.

19. Rojas-Villarraga A, Amaya-Amaya J, Rodriguez-Rodriguez A, Mantilla RD, Anaya JM. Introducing polyautoimmunity: Secondary autoimmune diseases no longer exist. Autoimmune Dis 2012;2012:254319.

20. Shiboski CH, Shiboski SC, Seror R, Criswell LA, Labetoulle M, Lietman TM, et al. 2016 American College of Rheumatology/European League Against Rheumatism classification criteria for primary Sjogren's syndrome: A consensus and data-driven methodology involving three international patient cohorts. Ann Rheum Dis 2017;76:9-16.

J Rheumatol 2019;46:665-6; doi:10.3899/jrheum.180392

Personal non-commercial use only. The Journal of Rheumatology Copyright $\subset$ $\subset$ 2019. All rights reserved 